\title{
Aplicación móvil para la captura de datos de inventario en plantaciones de eucalipto
}

\author{
Mobile application for capturing inventory data in eucalyptus plantations

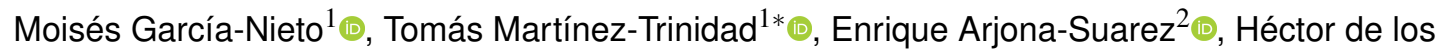

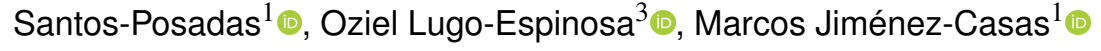 \\ ${ }^{1}$ Colegio de Postgraduados, Campus Montecillo, Posgrado en Ciencias Forestales. Km. 36.5 Carr. México-Texcoco. Montecillo, CP. \\ 56230. Texcoco, Estado de México, México. \\ ${ }^{2}$ Colegio de Postgraduados, Campus Montecillo, Posgrado en Estadística. Km. 36.5 Carr. México-Texcoco. Montecillo, CP. 56230. \\ Texcoco, Estado de México, México. \\ ${ }^{3}$ Universidad Autónoma del Estado de México, Centro Universitario Texcoco. Av. Jardín Zumpango s/n. Fracc. El Tejocote, CP. 56265. \\ Texcoco, Estado de México, México. \\ *Autor de correspondencia: tomtz@colpos.mx
}

Nota científica recibido: 23 de mayo de 2018 aceptado: 27 de agosto de 2018

RESUMEN. Se construyó una aplicación móvil en una tableta digital para la captura de datos en campo, para facilitar el suministro de información al software encargado de estimar el inventario y el crecimiento-rendimiento maderable en plantaciones de eucalipto. La aplicación se elaboró con la metodología desarrollo de aplicaciones móviles (MDAM). La evaluación de calidad de la aplicación se efectuó bajo las normas ISO 9126 y 14598-5 con un cuestionario de seis atributos de calidad y 20 items que se estructuraron con una escala Likert aditiva de intervalo (excelente, buena, regular, deficiente y mala). El análisis de resultados se realizó bajo las modalidades de atributos de calidad y facilidad de uso. En ambas modalidades los resultados muestran que los promedios estuvieron en el rango bueno-excelente, por lo que es factible implementar una aplicación móvil para la captura de datos que cumplan las expectativas de calidad y funcionamiento de los usuarios del sistema.

Palabras clave: Atributos, calidad, dispositivo, Likert, modelado.

ABSTRACT. A mobile application was built into a digital tablet for the capture of field data to facilitate the supply of information to software responsible for estimating the inventory and timber yield-growth in eucalyptus plantations. The application was developed using the Mobile Application Development Methodology (MADM). The quality evaluation of the application was carried out under ISO 9126 and 14598-5 standards using a questionnaire consisting of six quality attributes and 20 items that were structured with an additive Likert-type interval scale (excellent, good, fair, deficient and poor). The analysis of results was carried out under the modalities of quality attributes and ease of use. In both modalities the results show that the averages were in the good-excellent range, so it is feasible to implement a mobile application for data capture that meets the quality and performance expectations of system users.

Key words: Attributes, quality, device, Likert, modeling.

\section{INTRODUCCIÓN}

Las plantaciones forestales comerciales proporcionan una alternativa para reincorporar áreas degradadas y que aportan utilidad económica a las personas que optan por establecerlas. El manejo de plantaciones requiere de una diversidad de información que indique las existencias actuales, su incremento y rendimiento o productividad, y las rela- ciones intrínsecas entre los diversos componentes de los ecosistemas (Návar 2010). El éxito de una plantación se basa en el aumento continuo de sus dimensiones: altura, diámetro, área basal y volumen maderable que determina su crecimiento en un periodo de tiempo específico. La cuantificación frecuente de estas variables se requiere para aplicar un manejo silvícola, que es importante para promover el crecimiento de los árboles para obtener un mayor 
volumen maderable (Pérez et al. 2012). Por lo que es vital estar revisando de forma periódica las condiciones del recurso, por medio del monitoreo; para conocer el comportamiento de la plantación a través del tiempo (Ledo et al. 2012).

La estimación del volumen maderable para una plantación implica, definir con anticipación las variables que se van a medir y sus estimadores, lo que hará que se facilite el trabajo en campo y proporcione información precisa y confiable al proceso (Rojo et al. 2004). En este sentido, la obtención de los datos en campo es una tarea laboriosa; por lo que la recopilación de los mismos debe ser confiable para favorecer un aprovechamiento óptimo y realizar una buena planeación que determine cuándo, dónde y cuánta madera cortar; además de conocer y controlar variables como el volumen del inventario, las tasas de crecimiento y los datos dasométricos (Chiari et al. 2008).

La forma más común para recopilar datos dasométricos para un inventario, es mediante el uso de formatos en papel por medio del registro de los valores de las variables, para luego capturarlos y procesarlos (Soto-Cervantes et al. 2016, Mollinedo et al. 2016). Sin embargo, el avance tecnológico ha permitido incorporar diferentes dispositivos analógicos y digitales para cumplir esta tarea, desde el uso de la percepción remota (Ortiz-Reyes et al. 2015, TorresVivar et al. 2017), hasta la utilización de dispositivos móviles con la finalidad de que el proceso de captura sea más fácil y eficiente (Hoyos y Rincón 2014). En consecuencia, se tiene una apropiada definición de variables, medición y obtención de datos, que representa un cierto grado de éxito para los posteriores procesos. Por lo anterior, el objetivo fue construir y evaluar una aplicación digital que capture y almacene información para el inventario de plantaciones comerciales del género Eucalyptus, con un dispositivo móvil para facilitar la obtención de datos en campo.

\section{MATERIALES Y MÉTODOS}

\section{Área de estudio}

El trabajo se realizó con información de la empresa FOMEX, propietaria de 5896 ha de eucalipto en el municipio de Las Choapas, Veracruz, México, ubicadas en los $17^{\circ} 55^{\prime}$ LN y $94^{\circ} 06^{\prime}$ LO (García-Nieto et al. 2017). El clima para la zona es cálido categoría (Am), con temperatura media anual de $27^{\circ} \mathrm{C}$, abundantes lluvias en verano y principios de otoño (Rzedowski 2006).

\section{Datos dasométricos y atributos generales}

Los datos capturados con la aplicación móvil para cada árbol presente en el sitio levantado fueron: predio, orden, número de sitio, fecha, fuente de germoplasma, coordenada de parcela, fecha de plantación, responsable, número de árbol, diámetro normal, altura total y observaciones de la condición del árbol. Los valores válidos de los atributos generales (predio, órden, parcela, fecha del levantamiento, fecha de plantación, número de parcela o sitio, coordenadas, responsable, fuente del germoplasma y observaciones) se cargaron previamente en la base de datos de la aplicación para permitir la facilidad de captura.

\section{Atributos de calidad y escala Likert}

Se evaluaron los atributos de funcionalidad, confiabilidad, portabilidad, eficiencia, mantenibilidad, y usabilidad; que corresponden al uso generalizado en la medición de la calidad de software basado en las normas ISO 9126 y 14598-5. El tipo de escala se seleccionó en lugar de una escala ordinal, por proporcionar más información, debido a que es apropiada para medidas numéricas de tendencia central como de dispersión. El requisito de proporcionalidad subjetiva se cumple utilizando los valores: mala, deficiente, regular, buena y excelente. Para facilitar la interpretación de los resultados se utilizaron valores numéricos enteros -2, -1, 0, 1, 2 para representar los valores de la variable.

\section{Software y hardware}

En las etapas de análisis y diseño se recurrió al software Edraw Max ${ }^{\circledR}$ (EdrawSoft 2009), la base de datos se realizó con el gestor SQLite ${ }^{\circledR}$ (Hipp 2013) y la interfaz gráfica de desarrollo en Sqlitestudio ${ }^{\circledR}$ (Salawa 2013). Para la fase de desarrollo la codificación se efectuó con la interfaz gráfica AndroidStu- 
dio IDE ${ }^{\circledR}$ (NetBeans 2013). En lo que corresponde al hardware, para la implementación de la interfaz se utilizó una laptop con procesador Intel ${ }^{\circledR}$ Core $^{\mathrm{TM}_{2}}$ Duo CPU T6400 a $2.00 \mathrm{GHz}$, memoria RAM de 4.00 $\mathrm{GB}$, sistema operativo de 32 bits y una tableta digital ICONIA TAB A510 con sistema operativo Android ${ }^{\text {TM }}$ con procesador móvil NVIDIA ${ }^{\circledR}$ Tegra $^{\text {TM }} 3$ con CPU Quad-Core ARM ${ }^{\circledR}$ Cortex $^{\text {TM }}$-A9, memoria flash de $32 \mathrm{~GB}, 1 \mathrm{~GB}$ de RAM, GPS, Wi-Fi IEEE $802.11 \mathrm{~b} / \mathrm{g} / \mathrm{h}$, Bluetooth ${ }^{\circledR} 2.1+E D R$, dispositivo USB, batería recargable de polímeros de litio:9800 mAh (36,26 Wh), pantalla 10,1" WXGA (1280 x 800) TFT 262K LCD.

\section{Construcción de la aplicación}

La interfaz se creó con la Metodología para el Desarrollo de Aplicaciones Móviles (MDAM) y el Lenguaje Unificado de Modelado (UML). La aplicación MDAM es una metodología construida bajo cinco fases (análisis, diseño, desarrollo, pruebas de funcionamiento y entrega) y dieciséis tareas, distribuidas entre las fases de la siguiente manera: análisis (obtención de requerimientos, clasificación de requerimientos y personalización del servicio), diseño (definición del escenario, estructuración del software, definición de tiempos y asignación de recursos), desarrollo (codificación, pruebas unitarias, documentación del código y codificación de ayuda), pruebas de funcionamiento (emulación y simulación, dispositivos reales y evaluación) y entrega (manuales y distribución) (Gasca et al. 2014). El leguaje unificado de modelado (UML), tiene un papel esencial en la metodología, ya que se utiliza en las fases de análisis y diseño para describir la estructura y funcionamiento del software.

\section{Análisis de la aplicación}

Con las entrevistas realizadas con la empresa, se obtuvieron los requisitos de la aplicación. En primera instancia, se registraron los problemas que se tienen con el proceso actual (Figura 1). El personal captura los datos en campo con un formato en papel y refiere que por las condiciones de campo, su uso incorrecto propicia pérdida o alteración de los datos, y la duplicación del trabajo (primero recopilar y después capturar). Posteriormente, se obtuvieron los requisitos de la empresa, que definió que el proceso debería de ser más eficiente, para evitar los errores antes mencionados. Para lo cual, se propuso usar las tecnologías móviles, para facilitar los procesos de captura y almacenamiento. Por último, se establecieron los requerimientos funcionales de la aplicación de manera conjunta con los usuarios de la empresa considerando las siguientes tareas: captura de datos, almacenamiento (guardar, eliminar) y las siguientes condiciones: facilidad de uso y diseño sencillo; la primera de ellas, sugiere que la manipulación de la aplicación, en lo posible, no permita errores de entrada y la segunda propone, un orden lógico de los datos y una agradable presentación de la aplicación.

\section{Diseño de la aplicación}

La primera tarea realizada fue definir el escenario. Para este tipo de aplicación se requirió de un escenario fuera de línea, es decir, los procesos no necesitan estar conectados a una aplicación central o a un protocolo para funcionar, sino que estos se realizan de forma directa en el dispositivo móvil. El diseño de la aplicación se efectuó con el lenguaje unificado de modelado (UML), realizando diagramas de clases y actividades específicamente (Figura 2).

\section{Desarrollo de la aplicación}

La aplicación se construyó tomando en cuenta un formato de papel utilizado por los trabajadores para capturar los datos en campo. Las variables se eligieron para crear una interfaz consistente con la lógica del mismo y representarlo de forma semejante en el dispositivo móvil. Para la base de datos se consideró el modelo relacional con el gestor SQLite, dicho modelo se transformó a partir del diagrama de clases. La base de datos es la misma que utiliza el software que sirve para Estimar el Incremento y Crecimiento-Rendimiento Maderable (EICRM) (García-Nieto et al. 2017). La finalidad de utilizar la misma base de datos fue la compatibilidad de la información entre el dispositivo móvil y la computadora. 


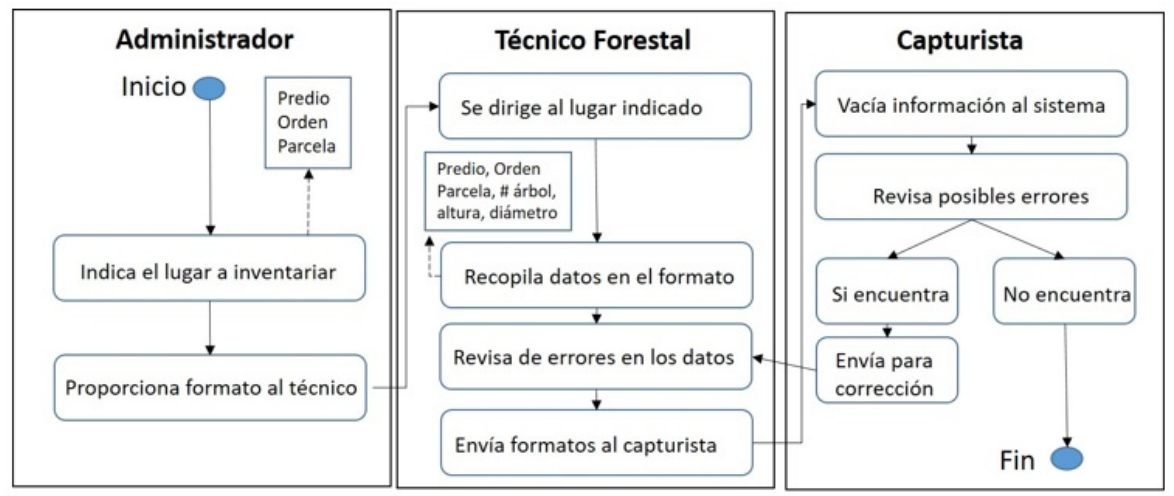

Figura 1. Proceso actual para el levantamiento de datos en campo

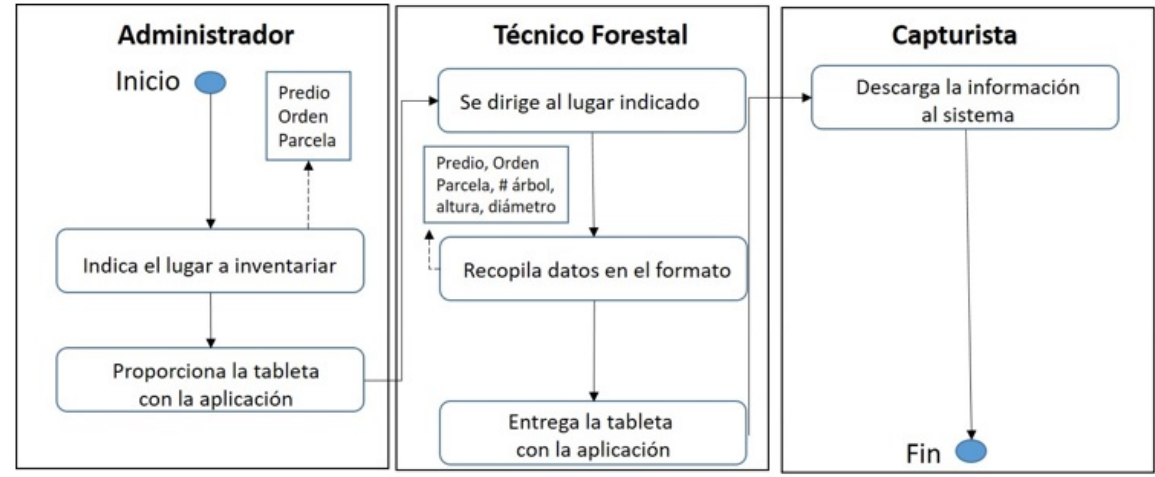

Figura 2. Proceso de levantamiento de datos en campo mediante la aplicación móvil.

\section{Pruebas de funcionamiento}

Se aplicaron las actividades de emulación y simulación, funcionamiento en dispositivo real, y evaluación de la aplicación. Para la primera tarea se realizan pruebas simulando el escenario y emulando el dispositivo móvil, explorando todas las utilidades y funciones de la aplicación, introduciendo diferentes datos, inclusive erróneos, para medir la funcionalidad y el nivel de robustez de la aplicación, de forma constante durante todo el proceso de codificación. El funcionamiento en la tableta digital se efectuó al final de la codificación, el cual consistió en instalar la aplicación en la tableta digital y manipularla para comprobar si existían o no errores, y si el funcionamiento era el óptimo. La evaluación se realizó con la metodología propuesta por Benítez y Ramírez (2013), la cual se basa en las normas ISO 9126 y 14598-5, que evalúan la calidad de las tecnologías flexibles mediante criterios externos e internos a medir. Considerando un muestreo dirigido a expertos, el cuestionario se aplicó a 10 usuarios finales con base a dos criterios: 1) que tuvieran conocimientos del uso de tabletas digitales, y 2) que conocieran el significado de los datos que se piden en la aplicación; apoyados en diseño de encuesta del trabajo de Marcos et al. (2006). Los resultados obtenidos de los 10 cuestionarios, se analizaron mediante estadística descriptiva para valores de intervalo con base en los factores y escalas definidos.

\section{RESULTADOS Y DISCUSIÓN}

Las variables de captura de datos en la aplicación móvil a través del lenguaje Unificado de Modelado, fueron las mismas variables que se registran en el formato de papel del inventario de 
la empresa FOMEX, mismas que se ingresaron a la base de datos del software para estimar el inventario y el crecimiento-rendimiento maderable en las plantaciones de eucalipto, para tener compatibilidad entre la aplicación y el software. El tipo de lenguaje utilizado fue esencial en la fase de diseño y estructura del software (Gasca et al. 2014). Las variables de circunferencia a la altura del pecho y altura total se obtuvieron en campo; mientras que a las otras variables se accede por medio de la base de datos de la aplicación. Las variables fuente de germoplasma y condición del árbol se seleccionan de la aplicación de una lista de valores predeterminados, las primera puede adquirir dos valores: semilla o clon; la segunda, puede adquirir los valores: $\mathrm{B}$ (bifurcado), CQ(copa quebrada), $\mathrm{P}$ (plagado o enfermo), MP(muerto en pie), M(muerto o derribado), I(inclinado pero vivo). La correcta caracterización de las variables favorece la comprensión en el proceso de tomas de datos de una plantación comercial (García-Nieto et al. 2017). Las acciones que se pueden realizar en la interfaz son: seleccionar (predios, órdenes y parcelas) así como guardar y eliminar datos (Figura 3); para luego compartirlos al software EICRM para realizar los cálculos del inventario (Figura 4).

La elaboración de aplicaciones promueve un sistema de codificación simple para la toma de datos en campo (Hoyos y Rincón 2014). La aplicación generó los siguientes archivos: src (carpeta que contiene el código fuente de la aplicación), gen (carpeta que contiene el código generado automáticamente por el SDK (Software Development Kit - Kit de desarrollo de software)), conjunto de herramientas y programas de desarrollo (permite al programador crear aplicaciones), Android xx (genera el código JAR de la API seleccionada), Android dependencies (librerías asociadas al proyecto), assets (carpeta que contiene ficheros usados por la aplicación), bin (carpeta que compila el código generando un archivo con la extensión .apk), libs (código JAR con librerías que se pueden usar en el proyecto), res (carpeta que contiene los recursos usados por la aplicación), AndroidManifest.xml (fichero que indica las actividades, intenciones, servicios y proveedores de la aplicación), y defaultpropertis (fichero generado automáticamente por el SDK).

En lo referente a la calidad del software, los resultados de la prueba de calidad demuestran que el valor máximo de las medias correspondió al factor de funcionalidad (2.750) y el valor mínimo (2.250) para el factor de usabilidad; mientras que para la desviación estándar el valor mínimo correspondió al factor de confiabilidad y el máximo al factor de eficiencia. Cabe indicar que los cuatro factores anteriores estuvieron en el rango de bueno a excelente. Debido a que en la escala Likert de intervalo es aditivita por promedio de los seis atributos de calidad, se obtuvo que la aplicación móvil presenta una calidad de rango de buena a excelente. La calidad de las aplicaciones de software es un factor importante, ya que una de sus finalidades es mejorar y facilitar las operaciones internas en las empresas o procesos para eficientar las gestiones de manera práctica (Benítez y Ramírez 2013).

Es factible que al integrar al usuario en la evaluación se incremente la calidad del software de forma significativa, en la mayoría de los factores propuestos (Amasaki et al. 2005). Incluso, factores como conformidad, exactitud, seguridad, comprensibilidad, facilidad de aprendizaje, operabilidad y tiempo de respuesta pueden tener un nivel de calidad buena; mientras que factores como madurez, tolerancia a fallas, recuperabilidad y adaptabilidad pueden tener una calidad aceptable. Por lo que todos los elementos evaluados cumplieron la prueba de calidad. Al respecto, Benítez y Ramírez (2013) reportan un nivel de excelencia y alto cumplimiento para los factores: eficiencia, portabilidad, funcionalidad, usabilidad, fiabilidad y mantenibilidad de la herramienta.

La evaluación de la aplicación por parte de los usuarios registró que cuatro consideraron la aplicación como excelente, tres aceptable y tres buena con respecto a la media. En este sentido, la evaluación hecha por los usuarios finales a la interfaz determinó que $70 \%$ la califique de excelente a buena y $30 \%$ de calidad aceptable a buena. El promedio de la media de los diez usuarios, indica que la calidad de la aplicación está en el rango de buena a exce- 


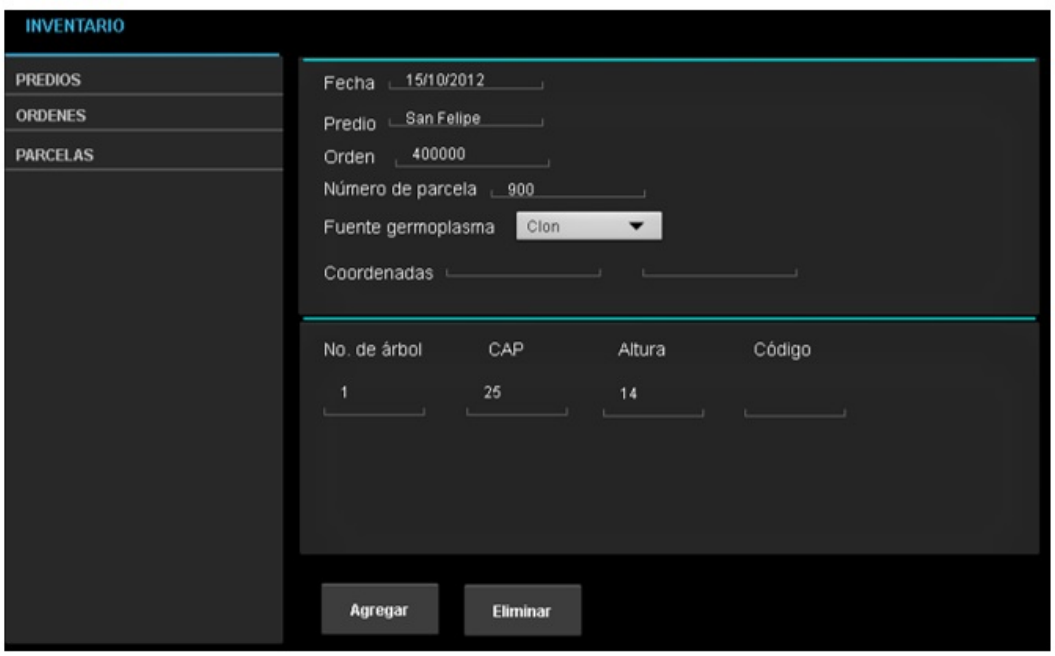

Figura 3. Interfaz del dispositivo móvil (tableta). Incluye las variables a capturar y las acciones para almacenar la información en la base de datos.

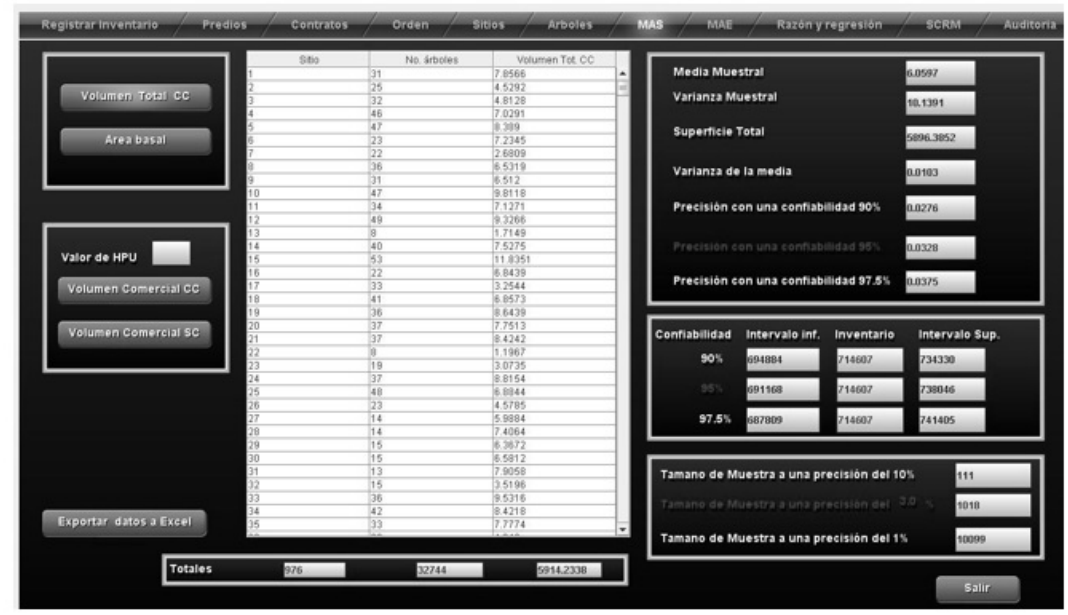

Figura 4. Pantalla del módulo de estimación del inventario del software EICRM en la que se observan los resultados obtenidos por los estimadores del muestreo aleatorio simple.

lente. El desarrollo de aplicaciones para proveer servicios móviles, difiere del desarrollo de software tradicional en muchos aspectos, lo que provoca que las metodologías usadas para estos entornos móviles, también difieren del software clásico (Gasca et al. 2014). La creación de las aplicaciones requieren la constante vinculación con los modelos de calidad que permitan evidenciar los niveles de excelencia de los productos e instrumentos de medición incluyendo variables, indicadores y factores que califiquen cada uno de los atributos que lo conforman (Benítez y
Ramírez 2013). Al respecto, Greiner et al. (2014) indican que la gestión cuantitativa de procesos, proporciona una visión del grado de cumplimiento de metas, así como un conocimiento cuantitativo, medible y determinable, cuyo rendimiento afecta de forma significativa el logro de los objetivos del proyecto y la satisfacción de los clientes.

Es factible generar una aplicación móvil de buena a excelente en una tableta digital para reducir la duplicación de trabajo y error de ilegibilidad de datos, debido a la facilidad de opciones múltiples 
existentes e incorporación de corrección de errores. Además, la aplicación reduce la duplicación del trabajo debido a que no se tiene que capturar de nuevo la información. La evaluación a través de los atributos de calidad estableció que la aplicación generada satisface las necesidades esenciales requeridas.

\section{AGRADECIMIENTOS}

Al Consejo Nacional de Ciencia y Tecnología y la Empresa Forestaciones Operativas de México, S.A. de C.V.

\section{LITERATURA CITADA}

Amasaki S, Yoshitomi T, Mizuno O, Takagi Y, Kikuno T (2005) A new challenge for applying time series metrics data to software quality estimation. Software Quality Journal 13: 177-193.

Benítez GJP, Ramírez RAL (2013) Evaluación de la calidad sobre la aplicación móvil fiscal para el cálculo de la PTU en empresas Mexicanas. Revista Iberoamericana para la Investigación y el Desarrollo Educativo 3: 219-236.

Chiari R, Carrero OE, Jerez M, Quintero MA, Stock J (2008) Modelo preliminar para la planificación del aprovechamiento en plantaciones forestales industriales en Venezuela. Interciencia 33: 802-809.

EdrawSoft (2009) Edraw Max 5 System for Windows. Versión 5.0. Sheung Wan, HongKong. Edrawsoft. Software de cómputo.

García-Nieto M, Martínez-Trinidad T, De los Santos-Posadas H, Lugo-Espinoza O, Acosta-Mireles M, ArjonaSuárez E, et al. (2017) Aplicación del proceso unificado en el desarrollo de un software que estima el inventario y el crecimiento-rendimiento maderable en plantaciones de eucalipto. Madera y Bosques 23: 163-178.

Gasca MMC, Camargo AL, Medina DB (2014) Metodología para el desarrollo de aplicaciones móviles. Tecnura 18: 20-35.

Greiner C, Dapozo G, Acosta J, Chiapello J (2014) Medición de software como aporte a la gestión cuantitativa de proyectos. In: RedUNCI (ed) XVI Workshop de Investigadores en Ciencias de la Computación. Ushuaia, Argentina. pp: 580-585.

Hipp R (2013) SQLite RDBMS system for Windows. Versión 3.7.16.1. Charlotte, NC. EUA:HWACI. Software de cómputo.

Hoyos MC, Rincón VO (2014) Uso de dispositivos móviles para la captura de datos en campo con formularios electrónicos a través del programa Cybertracker. Revista Palmas 35: 127-136.

Ledo A, Condés S, Montes F (2012) Revisión de índices de distribución espacial usados en inventarios forestales y su aplicación en bosques tropicales. Revista Perú Biología 19: 113-124.

Marcos MC, Mesa B, Ortega M, Benmakhlouf H, Dwelle P, et al. (2006) Evaluación de la usabilidad en sistemas de información terminológicos online. Hipertext.net, Universitat Pompeu Fabria. Barcelona, España. https://www.upf.edu/hipertextnet/numero-4/usabilidad.html. Fecha de consulta: 24 de febrero de 2017.

Mollinedo GMS, Herrera-M MA, Muñoz-S F (2016) Caracterización del crecimiento de plantaciones jóvenes de teca (Tectona grandis Linn f.) y estimación de curvas de índice de sitio en el área septentrional de la República de Guatemala. Madera y Bosques 22: 89-103.

Návar C (2010) Los bosques templados del estado de Nuevo León: el manejo sustentable para bienes y servicios ambientales. Madera y Bosques 16: 51-69. 
NetBeans (2013) Sun Microsystems/Oracle Corporation. Netbeans IDE 7.4. System for Windows. Redwood Shores, CA, EUA: Oracle Corporation. Software de cómputo.

Ortiz-Reyes AD, Valdez-Lazalde JR, De los Santos-Posadas HM, Ángeles-Pérez G, Paz-Pellet F, MartínezTrinidad T (2015) Inventario y cartografía de variables del bosque con datos derivados de LiDAR: comparación de métodos. Madera y Bosques 21: 111-128.

Pérez GG, Domínguez DM, Martínez ZP, Etchevers BJ (2012) Caracterización dasométrica e índice de sitio en plantaciones de caoba en Tabasco, México. Madera y Bosques 18: 7-24.

Rojo A, Rodríguez R, Rodríguez F, Castedo F, Grandas JA (2004) Dispositivos experimentales permanentes de gestión forestal sostenible para el estudio de modelos de crecimiento forestales en Galicia. Cuaderno de la Sociedad Española de Ciencias 18: 255-260.

Rzedowski J (2006) Vegetación de México. 1ra. Edición digital, Comisión Nacional para el Conocimiento y Uso de la Biodiversidad. México. 504p.

Salawa P (2013) SQLiteStudio system for Windows. (Versión 2.1.5). Kraków, Poonia: SalSoft Pawal Salawa. Software de cómputo.

Soto-Cervantes JA, López-Sánchez CA, Corral-Rivas JJ, Wehenkel CA, Álvarez-González JG, Crecente-Campo F (2016) Desarrollo de un modelo de perfil de copa para Pinus cooperi Blanco en la UMAFOR 1008, Durango, México. Revista Chapingo Serie Ciencias Forestales y del Ambiente 21: 179-192.

Torres-Vivar JE, Valdez-Lazalde JR, Ángeles-Pérez G, de los Santos-Posadas HM y Aguirre-Salado CA (2017) Inventario y mapeo de un bosque bajo manejo de pino con datos del sensor SPOT 6. Revista Mexicana de Ciencias Forestales 8: 25-43.

\section{INFORMACIÓN ADICIONAL}

Para más detalles sobre la aplicación contactar al primer autor al correo mogarni68@yahoo.com.mx 